Received: 2016.10 .13

Accepted: 2016.12 .21

Published: 2017.10 .20

\title{
Impact of Window Computed Tomography (CT) Parameters on Measurement of Inflammatory Changes in Paranasal Sinuses
}

Authors' Contribution:
A Study Design
B Data Collection
C Statistical Analysis
D Data Interpretation
E Manuscript Preparation
F Literature Search
G Funds Collection

\section{Maciej Cebula ${ }^{\text {ABGDEF }}$, Monika Danielak-Nowak ${ }^{\text {ABBi }}$, Sandra Modlińska ${ }^{\text {ABBF }}$}

Department of Radiodiagnostics and Invasive Radiology, School of Medicine in Katowice, Medical University of Silesia, Katowice, Poland

Author's address: Maciej Cebula, Department of Radiodiagnostics and Invasive Radiology, School of Medicine in Katowice, Medical University of Silesia, 16 Medyków Str., 40-752 Katowice, Poland, e-mail: mcebula@sum.edu.pl
Material/Methods:

Results:

Conclusions:

MeSH Keywords:

PDF file:

\section{Summary}

Background: In accordance with the European guidelines on rhinosinusitis and nasal polyps (EPOS 2012), CT is used as the main imaging modality for the assessment intensity of inflammatory lesions.

The aim of this study was to measure the differences in the assessment of inflammatory changes in the paranasal sinuses due to different parameters of width $(\mathrm{W})$ and length $(\mathrm{L})$ of the CT window.

A retrospective analysis included $44 \mathrm{CT}$ scans of the paranasal sinuses that were performed in adults. All studies were characterized by the presence of inflammatory changes in at least one of the sinuses.

Measurements of the same inflammatory lesions were performed sequentially with different CT windows. The results were statistically analyzed.

A statistically significant difference was observed between the average measurements that were performed with the use CT windows dedicated for the sinuses and head. A downward trend in the measured values and a shift towards soft tissue values was observed with decreasing window parameters.

A major cause of inaccurate examinations of pathological changes in the paranasal sinuses may be due to selection of unsuitable CT windows. Therefore, in order to avoid missing inflammatory lesions in the paranasal sinuses, it is reasonable to use CT windows dedicated for the sinuses or bones.

Dimensional Measurement Accuracy • Sinusitis • Tomography Scanners, X-Ray Computed

http://www.polradiol.com/abstract/index/idArt/901939

\section{Background}

The European guidelines on rhinosinusitis and nasal polyps (EPOS 2012) recommends endoscopy of the nasal cavities and CT of the paranasal sinuses as the standard methods for diagnosing inflammation of the paranasal sinuses. Visualization of inflammatory changes in at least one of the sinuses is necessary for diagnosis. This approach applies to both children and adults. In accordance with the above-mentioned guidelines, CT is used as the main imaging modality for the assessment of intensity of inflammatory lesions. It is also important for clinical decision-making and planning of functional endoscopic sinus surgery [1].

Given the crucial diagnostic role of $\mathrm{CT}$, a proper technique of this examination and precise measurements of inflammatory changes are extremely important.

The aim of this retrospective study was to measure differences in the assessment of inflammatory changes in the paranasal sinuses due to different parameters of width (W) and length $(\mathrm{L})$ of the CT window. The secondary aim was to analyze reproducibility of measurements of inflammatory 
changes in paranasal sinuses performed with different CT windows and by different raters.

\section{Material and Methods}

This retrospective study included 44 CT scans of paranasal sinuses performed in the year 2015 in the Department of Radiodiagnostics and Interventional Radiology, School of Medicine, Medical University of Silesia, Katowice with the LightSpeed Pro 32 (GE) scanner. The studied group consisted of 28 women aged $50.89 \pm 14.08$ years, and 16 men aged $51.25 \pm 17.21$ years. In all patients, the presence of inflammatory changes in at least one of the sinuses was noted. All scans were acquired with a standard protocol for the paranasal sinuses, in accordance with the procedures of calibrating [2]. The examinations were performed with $0.625 \mathrm{~mm}$ slice thickness, in the supine position, without gantry tilt, and scanner settings were adjusted to the range of the scanned area $(250-300 \mathrm{~mA}, 120 \mathrm{kV})$.

Using a dedicated station (AW General Electric), three raters performed measurements of the same inflammatory lesions independently and at different times. The measurements were made on axial images in the following CT windows:

- Sinuses (W: 3000, L 500).

- Bone (In 2000, L 350).

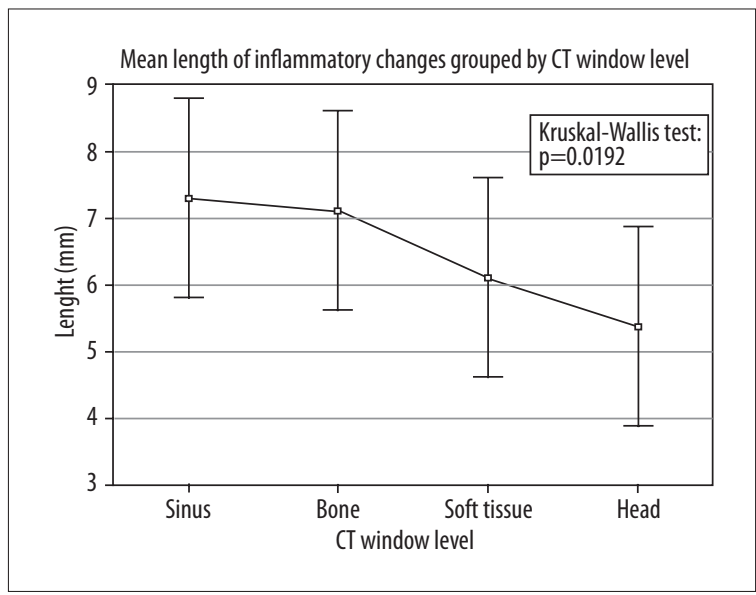

Figure 1. The mean and standard deviation of measurements of inflammatory lesions ( $\mathrm{mm}$ ) in all CT windows. Based on the Kruskal-Wallis test, the differences between the windows are statistically significant.
- Soft tissue (W: 400, L: 40).

- Head (W: 100 L: 35).

In order to minimize measurement errors, the values of individual raters were averaged for the analysis of measurement differences between different CT windows.

The database was created in Microsoft Excel (Microsoft), and statistical calculations were performed with Statistica 12.5 software (StatSoft).

The Shapiro-Wilk test was used to assess normality of distribution. In order to assess the significance of differences in the averaged measurements of changes between different CT windows, the Kruskal-Wallis and Dunn's tests were used. An accurate evaluation of measurement reproducibility with the R\&R analysis was not possible due to single measurements of each of the inflammatory lesions that were made by each rater. In order to compare the measurements of different raters that were made in particular CT windows, we used the Kruskal-Wallis test.

\section{Results}

Inflammatory changes were most commonly observed in the maxillary sinuses $(n=36)$. They were also present within the frontal sinus $(n=4)$, ethmoid sinus $(n=3)$, and sphenoid sinus $(\mathrm{n}=1)$. There was no significant correlation between sex, age, size, and location of the inflammation ( $p>0.05$ for all tests).

The distribution of average measurements of inflammatory lesions within the sinuses in each CT window is shown in Figure 1 and Table 1.

A statistically significant difference was observed in the average measurements between CT windows dedicated for sinuses and head $(\mathrm{p}<0.05)$. Based on the average measurements that were performed in each of the CT windows, a downward trend in the measured values and a shift towards the values assigned to soft tissues can be observed with decreasing window parameters.

The differences in measurements made by all raters in all CT windows are presented in Figure 2.

The differences between measurements performed by respective raters were not statistically significant in any of the tested windows $(p>0.05)$.

Table 1. Results of Dunn test between CT windows ( $p$ value).

\begin{tabular}{lcccc}
\hline & $\begin{array}{c}\text { Sinus } \\
\text { R: } \mathbf{1 0 1 . 5 2}\end{array}$ & $\begin{array}{c}\text { Bone } \\
\text { R: } \mathbf{9 8 . 0 0 0}\end{array}$ & $\begin{array}{c}\text { Soft tissue } \\
\text { R:83.250 }\end{array}$ & $\begin{array}{c}\text { Head R: } \\
\mathbf{7 1 . 2 2 7}\end{array}$ \\
\hline Sinus & - & 1.000 & 0.555 & 0.032 \\
\hline Bone & 1.000 & - & 1.000 & 0.082 \\
\hline Soft tissue & 0.555 & 1.000 & - & 1.000 \\
\hline Head & 0.032 & 0.082 & 1.000 & - \\
\hline
\end{tabular}

Kruskal-Wallis test result $p=0.019$. 


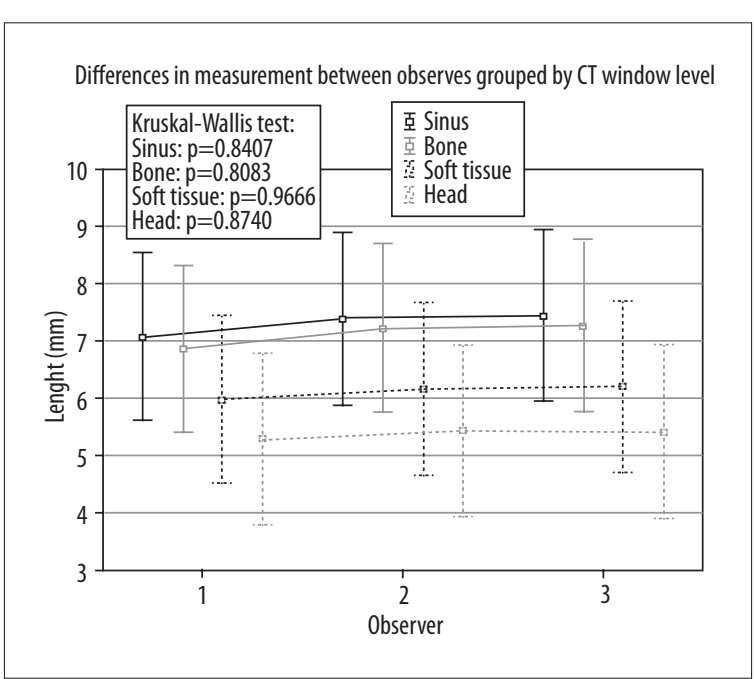

Figure 2. The mean and standard deviation of measurements of inflammatory lesions $(\mathrm{mm})$ in all CT windows, for each of rater. Based on the Kruskal-Wallis test, the differences between observers are not statistically significant.

\section{Discussion}

Acute sinusitis occurs in around $1-2 \%$ of the general population, and chronic sinusitis may affect even $10 \%$ of the population in Europe [3]. Not only do these diseases affect the quality of life of patients, but they also cause an economic problem [4]. In the USA, sinusitis is responsible for over two million patient visits and medical costs of over two billion dollars annually [5]. In Poland, family doctors issue sick leaves in 95\% of patients with sinusitis, and the average duration of such a leave is 7.5 days [6]. Given such a large scale of the problem, all radiologists deal with examinations of patients with sinusitis.

There is no correlation between the severity sinusitis that can be assessed in computed tomography and the intensity of symptoms. However, the usefulness of various imaging methods in diagnosing sinusitis has been proven [7].

The current guidelines for the diagnosis and treatment of sinusitis do not specify in detail what parameters should be evaluated on CT [1]. Radiological textbooks deliberate in great detail about the pathological changes that can be observed within the paranasal sinuses, but very rarely do they explain the measurement methodology. Since thickening of the mucous membrane greater than $3 \mathrm{~mm}$ is considered to be pathological, accuracy of measurement plays a vital role [8]. It is possible to overlook inflammatory changes, while analyzing CT examination of sinuses in the windows dedicated for soft tissues or head, instead of measuring the width of the mucous membrane in the bone or sinus window. We showed that the measurements are reproducible between different raters (Figure 2) and should correspond to the real size of the lesions. Notwithstanding technical factors such as the quality of the workstation screen and measurement reproducibility, the choice of CT window parameters is the only variable that should be considered as a possible source of misjudgment.

As regards X-rays that are performed for diagnosing sinusitis, they are routinely performed in the Waters projection (occipitomental, with open mouth) [5,9]. However, CT scans in the frontal plane are much more precise and more effective than X-ray images for the evaluation of complex pathologies involving the opening of maxillary sinuses, especially before potential endoscopic treatment [10].

In case of doubt as to the nature of lesions that are detected on CT, MRI of the paranasal sinuses is frequently able to narrow down the diagnosis. Strongly T2-weighted sequences are particularly useful, as they distinguish secretions in the paranasal sinuses from focal lesions [10]. Determination of the pathogen responsible for sinusitis is possible on CT, however, MRI has better sensitivity and specificity [11].

\section{Conclusions}

Due to an increasing number of cases of paranasal sinusitis, an accurate description of lesions in this region is important for making the diagnosis and planning treatment. Given a good reproducibility of measurement between independent raters, CT minimizes the risk of operatordependent errors. A major cause of inaccurate evaluations of pathological changes in the paranasal sinuses may be due to selection of unsuitable CT windows. Therefore, in order to avoid missing inflammatory lesions in the paranasal sinuses, it is reasonable to use CT windows dedicated for the sinuses or bones.

\section{Acknowledgements}

We would like to thank Professor Jan Baron, Head of Department of Radiodiagnostics and Invasive Radiology of Medical University of Silesia for his constant support, invaluable insight, and substantial guidance.

\section{Conflict of interests}

The authors do not have any disclosures or conflict of interests that they would like to declare in relation to their paper.

\section{References:}

1. Riechelmann $\mathrm{H}$, Giotakis A, Kral F et al: Acute rhinosinusitis in adults - EPOS 2012 Part II. Laryngorhinootologie, 2013; 92: 763-76

2. Statement of the Minister of Health of 10 November 2015 on the publication of the list of standard radiological procedures in the field of radiology - diagnostic imaging and interventional radiology (Acts. Office. Min. Of Healthy. From 2015., Pos. 78) [in Polish]

3. Fokkens W, Lund V, Mullol J et al: European position paper on rhinosinusitis and nasal polyps 2007. Rhinol Suppl, 2007; (20): $1-136$
4. Anand VK: Epidemiology and economic impact of rhinosinusitis. Ann Otol Rhinol Laryngol Suppl, 2004; 193: 3-5

5. Harnsberger HR, Osborn AG, Ross J, Macdonald A: Diagnostic and surgical imaging anatomy: Brain, head and neck. Spine, 2006; $1^{\text {st }}$ edition

6. Halvorsen PA, Wennevold K, Fleten N et al: Decisions on sick leave certifications for acute airways infections based on vignettes: A cross-sectional survey of GPs in Norway and Poland. Scand J Prim Health Care, 2011; 29(2): 110-16 
7. Stewart MG, Sicard MW, Piccirillo JF, Diaz-Marchan PJ: Severity staging in chronic sinusitis: are CT scan findings related to patient symptoms? Am J Rhinol, 1999; 13(3): 161-67

8. Tichenor WS: Sinus CT Scans, New York, New York. Avaliable from http://www.sinuses.com/ctscan.htm
9. Pruszyński B: Radiology - diagnostic imaging, X-ray, CT, ultrasound, MRI and nuclear medicine. Warsaw, 2011, $2^{\text {nd }}$ edition

10. Brant WE: Clyde Helms Fundamentals of diagnostic radiology. Warsaw, 2007, $1^{\text {st }}$ edition

11. Zinreich SJ, Kennedy DW, Malat J et al: Fungal sinusitis: Diagnosis with CT and MR imaging. Radiology, 1988; 169: 439-44 